\title{
Antibiotic resistance patterns among blood culture isolates in a Danish county 1981-1995
}

\author{
B. KRISTENSEN, H. H. SMEDEgAARD*, H. M. PEDERSEN*, M. F. ANDERSEN, J. F. DAHLERUP†, \\ H. T. SØRENSEN $\dagger+, B$. KORSAGER and H. C. SCHØNHEYDER
}

Departments of Clinical Microbiology and *Paediatrics, Aalborg Hospital, †Department of Medicine $V$, Aarhus University Hospital and $\ddagger$ Danish Epidemiology Science Centre at the Institute of Epidemiology and Social Medicine, University of Aarhus, Denmark

\begin{abstract}
All episodes of bacteraemia during a 15-year period (1981-1995) in the County of Northern Jutland, Denmark, were analysed with regard to antibiotic resistance. A total of 8840 isolates from 7938 episodes of bacteraemia was identified. Over time, no changes in bacterial aetiology were noted. Three isolates of Staphylococcus aureus were methicillin resistant $(0.2 \%)$ and six were gentamicin resistant $(0.4 \%)$. Among coagulase-negative staphylococci a $14 \%$ increase in resistance to penicillin was observed (95\% confidence intervals, CI: $2-26 \%$ ). Likewise, the frequency of resistance to methicillin, gentamicin and erythromycin increased, the corresponding figures being 38\% (CI: 26-50\%), 26\% (CI: 14-38\%) and 32\% (CI: 16-50\%), respectively, whereas a $14 \%$ decrease in resistance to streptomycin was recorded (CI: 4-24\%). A $20 \%$ (CI: $2-37 \%$ ) increase of coagulase-negative staphylococci resistant to three or more antibiotics was observed. The frequency of ampicillin resistance increased by $9 \%$ among Escherichia coli (CI: 4-13\%) and by 10\% (CI: 6-14\%) in all Enterobacteriaceae. Among Enterobacteriaceae the level of resistance to third-generation cephalosporins, carbapenems, aminoglycosides and fluoroquinolones remained low $(<1 \%)$. The frequency of resistance to three or more antibiotics remained fairly stable among Enterobacteriaceae, although a slight increase was noted among $E$. coli $(5 \%$; $C I$ : o-10\%) The recommended regimen for empirical antibiotic treatment in this region (a combination of penicillin $G$ or ampicillin and an aminoglycoside) provided an overall coverage of 94\% (CI: $94-95 \%$ ), although a slight decrease was noted at the end of the period. In conclusion, acquired antibiotic resistance was maintained at a low level compared with most other European countries and regions during the 15-year period studied.
\end{abstract}

\section{Introduction}

Prompt and adequate antibiotic treatment is a key factor in treating patients with severe infections including septicaemia. Knowledge of local antibiotic resistance patterns and potential changes is important when guidelines for empiric antibiotic therapy are made. One valid strategy for obtaining information on resistance patterns for individual hospitals and at the regional level is continuous surveillance of blood culture isolates. Consumption of antibiotics is an important determinant of the level of bacterial

Received 11 Feb. 1998; accepted 20 April 1998.

Corresponding author: Dr B. Kristensen. Present address: Department of Clinical Microbiology, Statens Serum Institute, Artillerivej 5, DK-2300 Copenhagen, Denmark. resistance $[1,2]$. In the County of Northern Jutland, Denmark, penicillin $\mathrm{G}$ or ampicillin in combination with an aminoglycoside have been the first-line antibiotics for patients with septicaemia for two decades. The aim of the present study was to examine the frequency of antibiotic resistance among blood culture isolates and analyse whether there have been any major changes in the patterns of resistance.

\section{Materials and methods}

The study comprised all bacterial isolates from positive blood cultures deemed clinically relevant in the County of Northern Jutland, Denmark (mean population 480000 inhabitants), during the period 1981-1995. Hospital service was free at all the county's one referral and 11 district hospitals. All blood cultures were 
investigated by the Department of Clinical Microbiology, Aalborg Hospital. For the years 1981-1991, all data were obtained retrospectively from laboratory records, whereas from 1992 onwards data were retrieved from a prospective computerised bacteraemia registry.

During the period 1981-1991, blood specimens were collected in Venoject ${ }^{\mathbb{B}}$ tubes that were transferred to the Department of Clinical Microbiology, Aalborg Hospital, for inoculation into multiple tubes of bacteriological media [3, 4]. In 1992, the blood culture system was changed to Colorbact ${ }^{\mathfrak{A})}$ (Statens Serum Institute, Copenhagen) [4,5]. Identification of isolates was according to standard practice $[6,7]$. Corynebacterium spp., Propionibacterium acnes, Bacillus spp., Flavobacterium spp. and Acinetobacter spp. were regarded as contaminants unless isolated from two or more separate blood culture sets. Identical isolates, i.e., isolates of the same species obtained from the same patient within 30 days, were counted only once.

Susceptibility testing was performed by tablet diffusion on Danish Horse Blood Agar (Statens Serum Institute) with NeoSensitabs ${ }^{(\overline{\mathrm{B}})}$ (Rosco Diagnostica, Taastrup, Denmark) following the manufacturer's criteria for zone-size interpretation [8-11]. The edges of the zones of inhibition were inspected carefully for microcolonies indicating $\beta$-lactamase production in Staphylococcus aureus [12]. If results of susceptibility testing were in conflict with intrinsic resistance mechanisms of enterobacterial species, e.g., ampicillin resistance in Klebsiella spp., or known patterns of cross-resistance (e.g., in staphylococci) [13], the former were over-ruled. For strains of $S$. aureus resistant to more than two antibiotics, confirmation of methicillin susceptibility was obtained by testing on nutrient agar with $\mathrm{NaCl} 7.5 \%$ which was incubated for 2 days at $30^{\circ} \mathrm{C}$ [14]. Monthly, quality control strains from national or international external quality assessment schemes were tested. For Enterobacteriaceae and most other gram-negative bacteria the following antibiotics were tested: ampicillin, mecillinam, piperacil- lin, imipenem, cefoxitin, cephalothin, cefuroxime, cefotaxime, streptomycin, gentamicin, netilmicin, ciprofloxacin, aztreonam, sulphonamides, tetracycline and chloramphenicol. For staphylococci and other gram-positive bacteria testing was done for penicillin, methicillin, cephalothin, streptomycin, gentamicin, tetracycline, sulphonamides, erythromycin, vancomycin and rifampicin. In assessing antibiotic resistance profiles, resistance to any number of cephalosporins was counted as one resistance character. Likewise, in staphylococci, methicillin and cephalosporin resistance was counted only once.

A $95 \%$ confidence interval (CI) is given for relative frequencies assuming a binomial distribution. CIs for a proportion and for difference between proportions were calculated by the method of Altman [15].

\section{Results}

A total of 7938 bacteraemias yielding 8840 bacterial isolates were recorded. Escherichia coli accounted for $31.7 \%$ of all isolates, whereas Klebsiella spp., $S$. aureus and coagulase-negative staphylococci accounted for $8.8 \%, 16.7 \%$ and $4.4 \%$, respectively. The distribution of bacterial species and groups was fairly constant throughout the period studied, although an increase in the isolation of anaerobes was noted (Table 1).

\section{Resistance in gram-negative species}

Ampicillin resistance was recorded in $41 \%$ of all Enterobacteriaceae at the start of the study period, but an increase of $10 \%$ (CI 6-14\%) was noted (Table 2), mainly due to a $9 \%$ (CI $4-13 \%$ ) increase of ampicillin-resistant E. coli. Simultaneously, resistance towards cephalothin decreased by $8 \%$ (CI 5-12\%) to $17.3 \%$. However, it should be noted that the susceptibility breakpoint for $E$. coli in Denmark $(<4 \mathrm{mg} / \mathrm{L})$ divides the homogeneous population of $E$. coli and, therefore, the significance of the observed decrease in cephalothin resistance is doubtful. Resistance towards

Table 1. The distribution of bacterial blood culture isolates in the County of Northern Jutland, Denmark 1981-1995

\begin{tabular}{|c|c|c|c|c|}
\hline \multirow[b]{2}{*}{ Bacteria } & \multicolumn{4}{|c|}{ Number $(\%)$ of isolates during } \\
\hline & $1981-1984$ & $1985-1988$ & $1989-1991$ & $1992-1995$ \\
\hline \multicolumn{5}{|l|}{ Gram-negative species } \\
\hline E. coli & $594(33.0)$ & $705(33.3)$ & $523(32.8)$ & $982(29.5)$ \\
\hline Klebsiella spp. & $137(7.5)$ & $204 \quad(9.6)$ & $151 \quad(9.3)$ & $288 \quad(8.7)$ \\
\hline Other Enterobacteriaceae & $192(10.7)$ & $228(10.8)$ & $115 \quad(7.2)$ & $252 \quad(7.6)$ \\
\hline Pseudomonas aeruginosa & $50 \quad(2.8)$ & $49 \quad(2.3)$ & 31 (1.9) & $70 \quad(2.1)$ \\
\hline Gram-negative anaerobes & $33 \quad(1.8)$ & $39 \quad(1.8)$ & $42 \quad(2.6)$ & $158 \quad(4.7)$ \\
\hline Other gram-negative species & $51 \quad(2.8)$ & $68 \quad(3.2)$ & $70 \quad(4.4)$ & $141 \quad(4.2)$ \\
\hline \multicolumn{5}{|l|}{ Gram-positive species } \\
\hline S. aureus & $353(19.6)$ & $346(16.3)$ & $285(17.9)$ & $489(14.7)$ \\
\hline $\begin{array}{l}\text { Coagulase-negative } \\
\text { staphylococci }\end{array}$ & 79 (4.4) & $87(4.1)$ & $49 \quad(3.1)$ & $171 \quad(5.1)$ \\
\hline Gram-positive anaerobes & $16 \quad(0.9)$ & $20 \quad(0.9)$ & $20 \quad(1.3)$ & $85 \quad(2.6)$ \\
\hline Other gram-positive species & $297(16.5)$ & $372(17.6)$ & $307(19.3)$ & $691(20.8)$ \\
\hline Total & 1802 & 2118 & 1593 & 3327 \\
\hline
\end{tabular}


Table 2. Antimicrobial resistance among Enterobacteriaceae from blood cultures in the County of Northern Jutland, Denmark 1981-1995

\begin{tabular}{|c|c|c|c|c|c|c|}
\hline \multirow{3}{*}{$\frac{\text { Antimicrobial agent }}{\text { Ampicillin }}$} & \multicolumn{6}{|c|}{ Number $(\%)$ of Enterobacteriaceae resistant during the period } \\
\hline & \multicolumn{2}{|c|}{$1981-1984$} & $1985-1988$ & \multicolumn{2}{|c|}{$1989-1991$} & \multirow{2}{*}{$\frac{1992-1995}{772(50.7)}$} \\
\hline & 378 & $(41.0)$ & $523(46.0)$ & 370 & $(47.0)$ & \\
\hline Mecillinam & 98 & $(10.6)$ & $136(12.0)$ & 64 & $(8.2)$ & $144(9.5)$ \\
\hline Piperacillin & $70^{*}$ & $(7.6)$ & $274(24.1)$ & 234 & $(30.1)$ & $568(37.3)$ \\
\hline Cephalothin & 236 & $(25.6)$ & $293(25.8)$ & 122 & $(15.7)$ & $263(17.3)$ \\
\hline Cefuroxime & $37^{*}$ & $(4.0)$ & $100 \quad(8.8)$ & 32 & (4.1) & $104(6.8)$ \\
\hline Cefotaxime & $5^{*}$ & $(0.5)$ & $13(1.1)$ & 5 & $(0.6)$ & $12(0.7)$ \\
\hline Imipenem & $1^{*}$ & $(0.1)$ & $2(0.2)$ & 1 & $(0.1)$ & $-t$ \\
\hline Ciprofloxacin & $-*$ & - & $4 \quad(0.4)$ & 1 & $(0.1)$ & $2(0.1)$ \\
\hline Gentamicin & 5 & $(0.5)$ & $5 \quad(0.4)$ & 5 & $(0.8)$ & $13 \quad(0.9)$ \\
\hline Streptomycin & 157 & $(17.0)$ & $236(20.8)$ & 138 & (17.5) & $-t^{\dagger}$ \\
\hline Aztreonam & 1 & $(0.1)$ & $16 \quad(1.4)$ & 5 & $(0.6)$ & $-{ }^{\dagger}$ \\
\hline Tetracycline & 188 & $(20.4)$ & $217(19.1)$ & 155 & $(19.7)$ & $-\dagger$ \\
\hline Sulphonamides & 173 & $(18.7)$ & $256(22.5)$ & 156 & $(19.8)$ & $-\dagger$ \\
\hline Chloramphenicol & 42 & $(4.6)$ & $57 \quad(5.0)$ & $5^{*}$ & $(1.0)$ & $-\dagger$ \\
\hline
\end{tabular}

${ }^{*}$ Not routinely tested in this period.

${ }^{\dagger}$ Data not recorded.

third-generation cephalosporins, carbapenems, fluoroquinolones and aminoglycosides was recorded in $<1 \%$ of all Enterobacteriaceae. During 1981-1991, a 7\% (CI $2-11 \%$ ) increase in tetracycline resistance was observed among $E$. coli whereas the level among all Enterobacteriaceae remained stable. In the same period, the frequency of resistance to three or more antibiotics remained fairly stable among Enterobacteriaceae (Table 3 ), although a slight increase was noted among $E$. coli (5\%; CI $0-10 \%)$.

Of 96 isolates of Haemophilus influenzae 7\% (CI $2-13 \%$ ) were ampicillin resistant. In Pseudomonas aeruginosa, resistance to aminoglycosides and piperacillin was rare $(0.5 \%$ and $3.0 \%$, respectively).

\section{Resistance in gram-positive species}

Among $S$. aureus isolates, the frequency of penicillin resistance was high, but remained stable (86-91\%), and resistance to methicillin $(0.2 \%)$ and gentamicin $(0.4 \%)$ was infrequent. Resistance to other antibiotics was negligible. Among the coagulase-negative staphylococci, the increase in penicillin resistance was $14 \%$ (CI 2-26\%) during the period 1981-1995 (Table 4). The corresponding figures for methicillin and gentamicin were $38 \%$ (CI 26-50\%) and 26\% (CI 14-38\%), respectively. During 1981-1991, the resistance to erythromycin increased by $32 \%$ (CI $16-50 \%$ ), whereas streptomycin resistance decreased by $14 \%$ (CI 4-24\%). A $20 \%$ (CI 2-37\%) increase in coagulase-negative

Table 3. Blood culture isolates resistant to three or more antibiotics in the County of Northern Jutland, Denmark 1981-1991

\begin{tabular}{lrrr}
\hline & \multicolumn{3}{c}{ Number (\%) of isolates resistant to three or more antibiotics } \\
\cline { 2 - 4 } & $1981-1984$ & $1985-1988$ & $1989-1991$ \\
\hline Bacterial isolate & $149(16.1)$ & $225(19.8)$ & $146(18.5)$ \\
\hline All Enterobacteriaceae & $113(19.0)$ & $168(23.8)$ & $125(23.9)$ \\
$E$. coli & $28(35.4)$ & $35(40.2)$ & $27(55.1)$ \\
Coagulase-negative staphylococci &
\end{tabular}

Table 4. Antimicrobial resistance among coagulase-negative staphylococci from blood cultures in the County of Northern Jutland, Denmark 1981-1995

\begin{tabular}{|c|c|c|c|c|}
\hline \multirow[b]{2}{*}{ Antimicrobial agent } & \multicolumn{4}{|c|}{ Number (\%) of coagulase-negative staphylococci resistant during } \\
\hline & $1981-1984$ & $1985-1988$ & $1989-1991$ & $1992-1995$ \\
\hline Penicillin & $54 \quad(68.4)$ & $71(81.6)$ & $43(87.8)$ & $141(82.5)$ \\
\hline Methicillin & $17(21.5)$ & $32(36.8)$ & $24(49.0)$ & $102(59.6)$ \\
\hline Gentamicin & $18 \quad(22.8)$ & $28(32.2)$ & $24(49.0)$ & $83(48.5)$ \\
\hline Streptomycin & $14 \quad(17.7)$ & $7 \quad(8.0)$ & $2(4.1)$ & $-t^{\dagger}$ \\
\hline Chloramphenicol & $9 \quad(11.4)$ & $14(16.1)$ & $7(14.3)$ & $--^{\dagger}$ \\
\hline Erythromycin & $18 \quad(22.8)$ & $21(24.1)$ & $27(55.1)$ & $--^{\dagger}$ \\
\hline Tetracycline & $18 \quad(22.8)$ & $13(14.9)$ & $9(18.4)$ & $-\dagger$ \\
\hline Sulphonamides & $9 \quad(11.4)$ & $9(10.3)$ & $3 \quad(6.1)$ & $--^{\dagger}$ \\
\hline Rifampicin & $0^{*} \quad(0.0)$ & $2(2.3)$ & $5(10.2)$ & $-^{\dagger}$ \\
\hline
\end{tabular}

* Not routinely tested in this period.

${ }^{\dagger}$ Data not recorded. 
staphylococci resistant to three or more antibiotics was observed (Table 3). No penicillin resistance was detected in 728 isolates of Streptococcus pneumoniae.

\section{Coverage}

During the 15-year study period, the recommended empiric antibiotic therapy for severe infections (i.e., septicaemia) consisting of penicillin $G$ or ampicillin combined with an aminoglycoside provided an overall coverage of $94 \%$ (CI 94-95\%). During 1981-1984, the coverage was $96 \%$, but during 1992-1995 the coverage was reduced to $92 \%$.

\section{Discussion}

In comparison with previous reports from this county $[16,17]$ as well as other reports from Scandinavia $[18,19]$, the present study found a fairly constant level of antimicrobial resistance among blood culture isolates during a 15 -year period. The most prominent trend was a moderate increase in ampicillin resistance among Enterobacteriaceae. This was reflected mainly in the increase of ampicillin-resistant $E$. coli. Ampicillin usage has been shown to be positively correlated to ampicillin resistance in $E$. coli [1]. In Denmark, ampicillin was the most frequently used $\beta$-lactam antibiotic when used for empiric antibiotic treatment in hospitals [4]. There are no exact statistics regarding antibiotic consumption at the hospitals, but Denmark had the lowest level of antibiotic consumption of all Scandinavian countries in the 1980s [20]. Resistance to third-generation cephalosporins, fluoroquinolones, gentamicin and imipenem was almost non-existent. This may reflect the regional policy of encouraging the use of penicillin $\mathrm{G}$ or ampicillin in combination with an aminoglycoside.

Among coagulase-negative staphylococci, the marked decrease in streptomycin resistance is probably due to the very limited consumption of streptomycin since the 1980s. Methicillin-resistant coagulase-negative staphylococci have been reported to be an indicator for antibiotic consumption [1]. In Denmark, the frequency of methicillin-resistant as well as multiresistant coagulase-negative staphylococci increased. This is a reminder of an ecological impact even of the moderate antibiotic usage at hospitals in this region. There is conflicting evidence as to whether the frequency of antimicrobial resistance among bacterial isolates is higher in nosocomial infections or in community-acquired infections [21,22]. From the data presented here it is not possible to distinguish between nosocomial and community-acquired bacteraemia. However, $>94 \%$ of all blood culture isolates were susceptible to the combination of a $\beta$-lactam and an aminoglycoside. The slight decrease in overall coverage was due to the slight increase of anaerobic gramnegative species naturally resistant to the empiric antibiotic therapy and the increased frequency of gentamicin-resistant coagulase-negative staphylococci. This emphasises the importance of monitoring antibiotic resistance and thus being able to optimise the use of antimicrobial agents; an issue which has been stressed by others [23].

To conclude, the level of acquired antibiotic resistance among blood culture isolates remained low, although there was a trend towards increasing resistance especially among coagulase-negative staphylococci and Enterobacteriaceae. The antibiotic policy remained unchanged during the survey period, but continuous monitoring remains necessary, as changing patterns of antibiotic resistance can necessitate adjustment of therapeutic guidelines.

This study was supported by grants from Obel's Foundation and the Danish Medical Research Council (NASTRA no. 9700677). The activities of the Danish Epidemiology Science Centre are financed by a grant from the Danish Research Foundation.

\section{References}

1. Møller JK. Antimicrobial usage and microbial resistance in a university hospital during a seven-year period. $J$ Antimicrob Chemother 1989; 24: 983-992.

2. Westh H. Erythromycin-resistant Staphylococcus aureus in Denmark. APMIS 1996; 104 Suppl 57: 1-37.

3. Bruun B, Sloth K, Bentzon MW, Frederiksen W. A study of bacteremias in Denmark from 1977-1978. Acta Pathol Microbiol Immunol Scand Sect B 1982; 90: 309-317.

4. Schønheyder HC, Højbjerg $T$. The impact of the first notification of positive blood cultures on antibiotic therapy. APMIS 1995; 103: 37-44

5. Cowan ST. Cowan and Steel's Manual for the identification of medical bacteria, 2nd edn. Cambridge, Cambridge University Press. 1974.

6. Prag J, Jensen J, Lebech K. Colorbact, a visually read blood culture system using darkening of haemoglobin in aerobic blood cultures as an early growth indicator, compared with Bactec $^{(\mathrm{R})}$ 6A and 7A. APMIS 1991; 99: 1089-1095.

7. Lenette EH, Balows A, Hausler WJ, Shadomy HJ (eds). Manual of clinical microbiology, 4th edn. Washington, DC, ASM Press. 1985.

8. Casal JB, Pedersen OG. Antimicrobial sensitivity testing using Neo-Sensitabs, 6th edn. Denmark, A/S Rosco. 1981.

9. Casal JB. Antimicrobial sensitivity testing using Neo-Sensitabs. 7th edn. Denmark, A/S Rosco. 1983.

10. Casal JB, Pringler N. Antimicrobial sensitivity testing using Neo-Sensitabs, 8th edn. Taastrup, Denmark, Rosco Diagnostica. 1985.

11. Casal JB, Pringler N. Antibacterial/antifungal sensitivity testing using Neo-Sensitabs, 9th edn. Taastrup, Denmark, Rosco Diagnostica. 1991.

12. Acar JF, Goldstein FW. Disk susceptibility test. In: Lorian V (ed) Antibiotics in laboratory medicine, 4th edn. Baltimore, Williams and Wilkins. 1996: 1-51.

13. Farmer JJ. Enterobacteriaceae: introduction and identification In: Murray PR, Baron EJ, Pfaller MA, Tenover FC, Yolken RH (eds) Manual of clinical microbiology, 6th edn. Washington, DC, ASM Press. 1995: 438-449.

14. Rosdahl VT, Frimodt-Møller N, Bentzon MW. Resistance to dicloxacillin, methicillin and oxacillin in methicillin-susceptible and methicillin-resistant Staphylococcus aureus detected by dilution and diffusion methods. APMIS 1989; 97: 715-722.

15. Altman DG. Practical statistics for medical research. London, Chapman and Hall. 1991.

16. Jensen KT, Scheel O. Antibiotikaresistens hos gram-negative bakterier fra bloddyrkninger. [Resistance to antibiotic gramnegative bacteria from blood cultures.] Ugeskr Lager 1988; 150: $2459-2462$. 
17. Jensen KT, Scheel O. Antibiotikaresistens hos gram-positive bakterier fra bloddyrkninger. [Resistance to antibiotics in grampositive bacteria from blood cultures.] Ugeskr Lager 1988; 150: $3056-3058$

18. Scheel O, Iversen G. Resistant strains isolated from bacteremia patients in northern Norway. Scand J Infect Dis 1991; 23: 599-605.

19. Walder M, Karlsson E, Nilsson B. Sensitivity of 880 blood culture isolates to 24 antibiotics. Scand J Infect Dis 1994; 26: 67-75.

20. Friis H, Vejlsgaard R. Antibiotikaforbrug i Norden. [Consumption of antibiotics in Scandinavia.] Ugeskr Lager 1986; 148 2853-2855
21. Ellner PD, Fink DJ, Neu HC, Parry MF. Epidemiological factors affecting antimicrobial resistance of common bacterial isolates. J. Clin Microbiol 1987; 25: 1668-1674.

22. McGowan JE, Hall EC, Parrott PL. Antimicrobial susceptibility in gram-negative bacteremia: are nosocomial isolates really more resistant. Antimicrob Agents Chemother 1989; 33: 18551859.

23. Goldmann DA, Weinstein RA, Wenzel RP et al. Strategies to prevent and control the emergence and spread of antimicrobialresistant microorganisms in hospitals. A challenge to hospital leadership JAMA 1996; 275: 234-240. 